\title{
Francisella philomiragia, bacteria asociada con altas mortalidades en salmones del Atlántico (Salmo salar) cultivados en balsas-jaulas en el lago Llanquihue
}

\author{
Francisella philomiragia, a bacteria associated with high mortalities \\ in Atlantic salmon (Salmo salar) cage-farmed in Llanquihue lake \\ H Bohle*, E Tapia, A Martínez, M Rozas, A Figueroa, P Bustos \\ Laboratorio de Diagnóstico y Biotecnología, ADL Diagnostic Chile Ltda., Puerto Montt, Chile.
}

\begin{abstract}
SUMMARY
Francisella philomiragia was isolated from Atlantic salmon cage-farmed in the Llanquihue lake with outbreaks of a granulomatous disease, with high rates of morbidity and an accumulated mortalities between $5 \%$ to $20 \%$. The isolates had $100 \%$ similarity with F. philomiragia ssp noatunensis or F. piscicida isolated in Atlantic cod, 99\% similarity with Francisella sp. detected in tilapia from Asia and Central America and $99 \%$ of similarity with the reference strain $F$. philomiragia through $16 s$ rDNA phylogenetic analysis.
\end{abstract}

Palabras clave: Francisella philomiragia, salmón del Atlántico, análisis filogenético.

Key words: Francisella philomiragia, Atlantic salmon, phylogenetic analysis.

\section{INTRODUCCIÓN}

A fines de la década de 1980, se detectó en Chile una nueva patología infecciosa cuyo agente causal presentaba morfología rickettsial (Bravo y Campos 1989) y fue denominado "unidentified agent". Este agente fue aislado por primera vez en una línea celular de embrión de salmón Chinook CHSE-214 (Fryer y col 1990) y posteriormente identificado como Piscirickettsia salmonis (Fryer y col 1992). La piscirickettsiosis ha sido una de las principales causas de mortalidad en salmónidos cultivados en las costas marinas del sur de Chile y ha significado grandes pérdidas económicas para la salmonicultura chilena. Sin embargo, a mediados de la década de los 1990 se logró aislar un segundo agente con morfología rickettsial en salmón del Atlántico cultivado en el lago Llanquihue (Cvitanich y col 1995), denominado "unidentified agent 2" (U2 o UA-2) para diferenciarlo de $P$. salmonis (Unidentified agent). Desde junio del 2006 planteles de salmón del Atlántico cultivado en balsas-jaulas en el lago Llanquihue presentaron altas tasas de morbilidad y mortalidad, con varios y simultáneos brotes de una enfermedad crónica de tipo granulomatosa principalmente evidenciada a nivel de bazo y que en todos los casos se detectó y se aisló un agente bacteriano con morfología rickettsial sugerente de U2 (Birkbeck y col 2007).

El análisis filogenético de las secuencias del gen 16s rDNA de los aislados reveló pertenecer a la familia Francisellaceae, la que fue descrita en el año 2005 por

Aceptado: 03.06.2009.

* Casilla 160, Puerto Montt, Chile; hbohle@adldiagnostic.cl
Sjøstedt y agrupa a bacterias dentro de la subclase gamma proteobacteria y tiene un solo género, Francisella. Este género tiene dos especies tipo, F. tularensis patógeno humano (Nano y col 1992) y F. philomiragia aislado desde fuentes de agua, ratas de río y de algunas infecciones accidentales en humanos (Hollis y col 1989). Adicionalmente, existe una gran cantidad y variedad de otras Francisella sp. de afiliación incierta y que han sido aisladas de diversas fuentes tales como garrapatas (Scoles 2004), del ambiente (Barns y col 2005) y de una amplia variedad de peces (Chen y col 1994, Mauel y col 2003, Nylund y col 2006). El análisis filogenético de los genes $16 \mathrm{~s} \mathrm{rDNA}$, espaciador intergénico (ITS), 23s rDNA, rpoB, fopA, groEL, lpnB y sdhA, reveló que los aislados pertenecen al grupo $F$. philomiragia y un $100 \%$ de similitud con $F$. philomiragia ssp noatunensis (Mikalsen y col 2007), formando parte del mismo grupo en conjunto con otras Francisellas aisladas de bacalao en Noruega, tilapia en Asia y América Central (Nylund y col 2007, Mikalsen y col 2007, Mauel y col 2007).

El presente estudio tiene como objetivo mostrar antecedentes generales de la patología, condiciones de aislamiento, fisiológicos, susceptibilidad y filogenéticos de los aislados bacterianos de Francisella philomiragia aislados de salmón del Atlántico.

\section{MATERIAL Y MÉTODOS}

PECES

Salmones del Atlántico con brotes de la enfermedad fueron capturados y trasladados al laboratorio de diagnóstico y biotecnología de ADL Diagnostic Chile Ltda. para su análisis clínico. Los peces presentaron un peso 
promedio de $60 \mathrm{~g}$ ( $30 \mathrm{a} 80 \mathrm{~g}$ ), con una mortalidad mensual acumulada entre 5 a $20 \%$ en la mayoría de los centros de cultivo ( 9 de 15 centros).

\section{ANÁLISIS BACTERIOLÓGICO, SUSCEPTIBILIDAD} ANTIMICROBIANA Y CULTIVO CELULAR

Tejidos de riñón, bazo, hígado, corazón, aletas y branquias de los peces afectados fueron cultivados bajo condiciones de completa asepsia en los medios bacteriológicos agar tripticasa de soya ${ }^{1}$, agar columbia suplementado con $5 \%$ sangre cordero $^{2}$, agar Mueller-Hinton ${ }^{1}$, Agar nutriente $^{1} 0 \%$ y $6 \% \mathrm{NaCl}$, agar $\mathrm{BHI}^{1}$ y agar Eugon ${ }^{1}$ suplementado con $0,1 \%$ L-cisteína ${ }^{3}, 1 \%$ glucosa $^{4}, 6 \%$ (v/v) yeastolate ${ }^{5}$ y $10 \%$ $(\mathrm{v} / \mathrm{v})$ suero fetal bovino 6 e incubados por 8 semanas a $18^{\circ} \mathrm{C}$ con monitoreo diario para la observación de crecimiento de colonias bacterianas. Desde las colonias bacterianas se realizó tinción $\mathrm{Gram}^{4}$ para evaluar la morfología y características tintoriales, además de las pruebas bioquímicas de oxidasa ${ }^{4}$ y catalasa. Adicionalmente, se determinó la sobrevivencia de los aislados en agar Eugon enriquecido y suplementado con diferentes concentraciones finales de $\mathrm{NaCl}^{4}(0,4 \%, 1 \%, 2 \%, 3 \%$ y $6 \%)$ a $18{ }^{\circ} \mathrm{C}$ por 8 semanas de incubación. Paralelamente, se evaluó el desarrollo de colonias de los aislados en agar Eugon enriquecido a diferentes temperaturas $(5,10,15,18,20,22,25,30$ y $37{ }^{\circ} \mathrm{C}$ ) durante 8 semanas de incubación. Se determinó la concentración inhibitoria mínima de los aislados para florfenicol $^{7}$, amoxicilina ${ }^{7}$, flumequina ${ }^{7}$, oxitetraciclina ${ }^{7}$ y ácido oxolínico ${ }^{7}$ según método de medición de susceptibilidad en dilución en caldo para bacterias aisladas de animales acuáticos (M49-A, CLSI 2006) usando como control E. coli ATCC 25922.

Riñones asépticamente tomados con tórula ${ }^{2}$ fueron inoculados en placas de 24 pocillos $^{8}$ con las líneas celulares CHSE-214 (Chinook salmo embryo) cultivado en MEM-10 5 , 2 mM L-glutamina ${ }^{5}$ y sin antibióticos. Otras líneas celulares también fueron inoculadas como SHK-1 (Salmo salar head kidney), BF-2 (Bluegill fry caudal trunk) en medio Leibovitz L- $15^{5}$ y en líneas celulares continuas desarrolladas en ADL Diagnostic Chile Ltda., correspondientes a RTR-2 (Rainbow trout renoma, 2005) y OKE-1 (Coho salmo embryo, 2003) cultivadas en Leibovitz L-15. Todas las líneas celulares fueron incubadas por 8 semanas a $15^{\circ} \mathrm{C}$ con monitoreo diario para la aparición de efecto citopático (ECP).
ANÁLISIS HISTOPATOLÓGICO Y MICROSCOPÍA ELECTRÓNICA

Trozos de riñón, bazo e hígado de los peces con lesiones granulomatosas fueron fijados en formaldehído al $10 \%$ en PBS (pH 7,2) para su análisis histopatológico. Las muestras procesadas fueron teñidas con hematoxilina-eosina de Harris y tinción Gram previo a su análisis.

Para el análisis por microscopia electrónica fue cosechado $1 \mathrm{ml}$ de suspensión celular de las líneas celulares que presentaron $50 \%$ CPE y se centrifugó a $3.000 \mathrm{rpm}$ por 10 minutos, posteriormente el sobrenadante fue eliminado y el botón celular fue resuspendido en glutaraldehído ${ }^{4}$ al $3 \%$ en PBS ( $\mathrm{pH} 7,2$ ). Las muestras fueron enviadas a la Universidad Prince Edward Island (UPEI) en la ciudad Charlottetown en Canadá para su procesamiento y análisis.

AMPLIFICACIÓN Y SECUENCIAMIENTO DE LOS GENES $16 \mathrm{~s}$ rDNA, ITS, 23s rDNA, rpoB, fopA, groEL, lpnB Y sdhA

El ADN genómico bacteriano fue extraído utilizando las columnas de extracción EZNA ${ }^{\circledR}$ tissue DNA kit ${ }^{9}$, siguiendo las instrucciones del fabricante. La amplificación por PCR de los genes fue llevada a cabo utilizando los partidores descritos en el cuadro 1. Las amplificaciones de PCR fueron ejecutadas en un termociclador T3 (Biometra, Inglaterra) en un volumen final de $50 \mu \mathrm{l}$, con una mezcla de $46 \mu$ que contiene $1 \mathrm{U}$ Taq polimerasa ${ }^{10}, 20 \mathrm{mM}$ Tris- $\mathrm{HCl}$ (pH 8,4), $50 \mathrm{mM} \mathrm{KCl,} \mathrm{0,2} \mathrm{mM} \mathrm{dNTP,} \mathrm{1,5} \mathrm{mM}$ $\mathrm{MgCl}_{2}, 0,2 \mu \mathrm{M}$ de cada partidor y $4 \mu \mathrm{l}$ de templado. El perfil térmico utilizado fue de: paso de denaturación inicial a $95^{\circ} \mathrm{C}$ por $3 \mathrm{~min} ; 30$ ciclos a $95^{\circ} \mathrm{C}$ por $40 \mathrm{seg}, 50{ }^{\circ} \mathrm{C}$ por $40 \mathrm{seg}, 72{ }^{\circ} \mathrm{C}$ por $1,5 \mathrm{~min}$, seguido por una extensión final a $72{ }^{\circ} \mathrm{C}$ por $5 \mathrm{~min}$. Los productos de PCR fueron analizados por electroforesis en gel de agarosa $2 \%$ y las bandas correspondientes purificadas con el kit comercial EZNA® Gel extraction kit $^{9}$ siguiendo las instrucciones del fabricante.

El ADN purificado fue secuenciado con el Kit BigDye ${ }^{\circledR}$ Terminator v3.1 $1^{11}$ en un analizador genético ABI PRISM 310 (Applied Biosystem, EUA) utilizando partidores descritos en el cuadro 1. Las secuencias obtenidas fueron analizadas y editadas con el software Sequencing Analysis v5.2 (Applied Biosystem, EUA) y luego publicadas en la base de datos pública GenBank (NCBI, EUA) con los siguientes números de acceso: EU931318 rpoB (261 pb), EU931319 groEL (702 pb), EU931320 sdhA (218 pb), EU931321 fopA (960 pb), EU931322 lpnB (252 pb) y EU931323 para $16 s$ ITS $23 s$ rDNA (1748 pb).

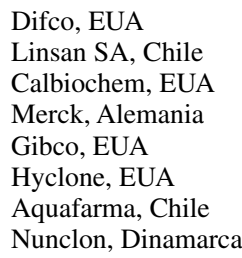

9 Omega Bio-Tek, EUA

10 Invitrogen, EUA

11 Applied Biosystems, EUA 
Cuadro 1. Conjunto de partidores usados para el secuenciamiento genético de F. philomiragia aislados de salmón del Atlántico. Set of primers used in the genetic sequencing of F. philomiragia isolated from Atlantic salmon.

\begin{tabular}{lcc}
\hline gen & sentido & antisentido \\
\hline sdhA & 5'-ACTTGTGCTGCGGCTGATAGAAC-3' & 5'-TACGACCTGCTCCACCAGTAGC-3' \\
fopA & 5'-CAGATCCAAATCATATTGAAACTC-3' & 5'-TGCTATTGCTTCTTTACCTTTAG-3' \\
lpnB & 5'-GTAACCTTGATAATCCTGATC-3' & 5'-GAACACTCTGATTGTCCAC-3' \\
groEL & 5'-TACTTTCTAAGCCTTGTTCAG-3' & 5'-AAGAGCATCATCCACACG-3' \\
$r p o B$ & 5'-GTTGAGAACCAGTTTAGAATTG-3' & 5'-CTTCAAAGCCTGCTCTATCACG-3' \\
$16 s+I T S+23 s$ rDNA set 1 & 5'-AACGGTAACAGGTCTTCG-3' & 5'-ACCAGGTAAGGTTCTTCG-3' \\
$16 s+I T S+23 s$ rDNA set 2 & 5'-CTTACCTGGTCTTGACATCC-3' & 5'-GCTTATCGCAGATTAACACG-3' \\
$16 s+I T S+23 s$ rDNA set 3 & 5'-GTGTTAATCTGCGATAAG-3' & 5'-GTTTCCCTTTCCACTACG-3' \\
\hline
\end{tabular}

\section{ANÁLISIS FILOGENÉTICO}

El análisis filogenético de los genes fue realizado por alineamientos múltiples de secuencias de especies bacterianas relacionadas para el gen $16 s$ rDNA (figura 1), con la información de los genomas de Francisella disponibles en GenBank para los genes $16 s+I T S+23 s$ rDNA (figura 2a) y el análisis multilocus con los genes constitutivos (Housekeeping) rpoB, groEL, $s d h A$, fopA y lpnB (figura 2b) con el software ContigExpress y AlignX (Vector NT $i$ v10.0, Invitrogen, EUA), editados y exportados a formato NEXUS con BioEdit 7.0.9.0 (Hall 1999). Los tres árboles filogenéticos obtenidos fueron construidos con el criterio de la regla de la mayoría (50\%) a partir de 1000 réplicas de bootstrap con el método de máxima similitud ejecutado en PAUP v4.0b10 (Swofford 2002) usando el proceso heurístico de búsqueda con la opción de bisección y recombinación (TBR) con el modelo HKY+G para el análisis del gen 16s rDNA, HKY para el análisis de los genes $16 s+I T S+23 s$ rDNA y $\mathrm{TrN}+\mathrm{G}$ para el análisis multilocus de los genes constitutivos, todos los modelos seleccionados por el software Modeltest (Posada y Crandall 1998).

\section{RESULTADOS}

\section{OBSERVACIONES DE CAMPO Y NECROPSIA}

Los peces afectados se caracterizaron por presentar anorexia, natación errática en la superficie y letargia. A la necropsia, mostraron aumento de tamaño del hígado, riñón y bazo, con múltiples focos blanquecinos en el hígado y bazo, con grasa perivisceral y vejiga natatoria congestiva y en los casos más severos con equimosis.

\section{ANÁLISIS BACTERIOLÓGICO, SUSCEPTIBILIDAD ANTIMICROBIANA Y CULTIVO CELULAR}

Los cultivos bacterianos en agar tripticasa de soya, agar columbia sangre, agar Mueller-Hinton, agar nutriente con y sin sal y agar BHI fueron negativos a las 8 semanas de incubación. Sin embargo, se observó desarrollo de colonias redondas de 1 a $2 \mathrm{~mm}$ de diámetro, de consistencia butirosa, convexas, brillantes, de color gris-verdoso sin pigmento difusible en agar Eugon, con crecimiento visible a los 12 días de incubación a $18{ }^{\circ} \mathrm{C}$. La tinción Gram de las colonias evidenció estructuras cocoides y cocobacilares Gram negativas dispuestas individuales, en pares y agrupaciones, con test de oxidasa negativo y reacción de catalasa débil. En el desafío a diferentes salinidades, los aislados se desarrollaron solamente entre $0,4 \%$ a $1 \%$ $\mathrm{NaCl}$, escasamente a $2 \%$ y no hubo desarrollo a $3 \%$ y $6 \%$. Adicionalmente, en el desafío a diferentes temperaturas, los aislados no se desarrollaron a $5{ }^{\circ} \mathrm{C}, 30^{\circ} \mathrm{C}$ y $37^{\circ} \mathrm{C}$ a las 8 semanas de incubación, el desarrollo fue lento a los $10{ }^{\circ} \mathrm{C}, 15^{\circ} \mathrm{C}$ y $25^{\circ} \mathrm{C}$ y abundante a los $18^{\circ} \mathrm{C}, 20^{\circ} \mathrm{C}$ y $22{ }^{\circ} \mathrm{C}$. Se evidenció desarrollo inicial a los 6 días a $22^{\circ} \mathrm{C}$ y 12 días a $18^{\circ} \mathrm{C}$ de incubación. Las concentraciones inhibitorias mínimas obtenidas fueron para florfenicol 1,0 $\mu \mathrm{g} /$ $\mathrm{ml}$, flumequina $0,25 \mu \mathrm{g} / \mathrm{ml}$, ácido oxolínico $0,25 \mu \mathrm{g} / \mathrm{ml}$, oxitetraciclina $0,5 \mu \mathrm{g} / \mathrm{ml}$ y amoxicilina $>64,0 \mu \mathrm{g} / \mathrm{ml}$.

En los cultivos celulares se observó efecto citopático en OKE-1 a las dos semanas de incubación, a diferencia de BF-2 donde se observó desprendimiento celular de la monocapa celular a los 12 días de incubación; SHK-1 presentó CPE y desprendimiento celular a los 20 días de incubación. El resto de las líneas celulares CHSE-214 y RTR-2 no presentaron efecto citopático o desprendimiento celular a las ocho semanas de incubación; sin embargo, a la tinción Gram y microscopía electrónica se observó la presencia del agente y cambio en la morfología celular ultraestructural en estas últimas líneas celulares.

\section{ANÁLISIS HISTOPATOLÓGICO Y MICROSCOPÍA ELECTRÓNICA}

El análisis histopatológico reveló una patología sistémica con degeneración celular y necrosis ampliamente diseminada a través del hígado, bazo y riñón. Los bazos presentaron degeneración del tejido conectivo y con esplenocitos con núcleos picnóticos y cariorréxicos. Los leucocitos y en particular los macrófagos fueron observados como células 


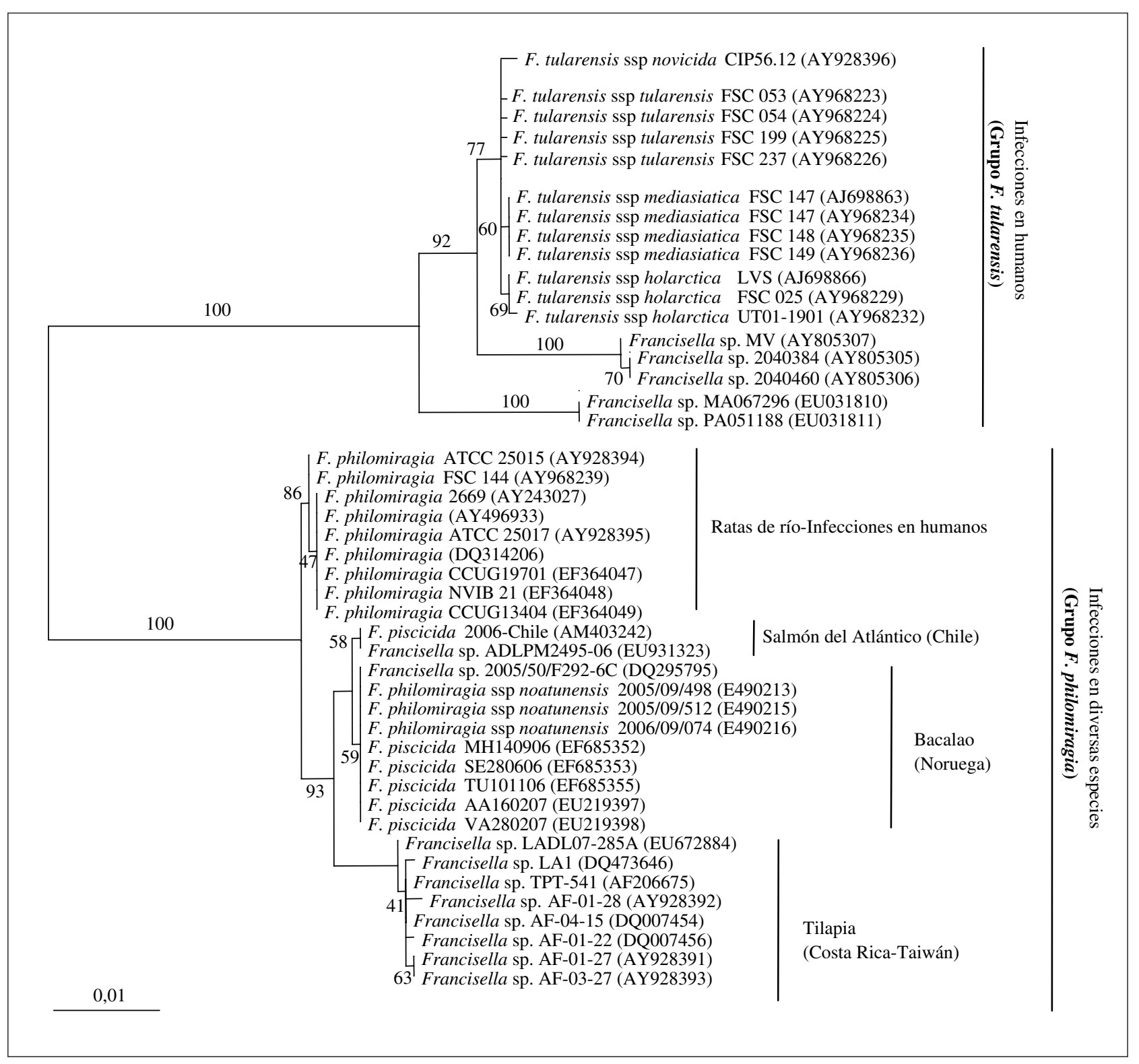

Figura 1. Relación filogenética de los aislados de Francisella salmón del Atlántico en Chile y otras Francisellas aisladas de diferentes fuentes como bacalao, tilapia, garrapatas y mamíferos en base a las secuencias del gen 16s rDNA. El árbol filogenético fue ruteado con el grupo F. tularensis.

Phylogenetic relationship of the isolates of Francisella from Atlantic salmon in Chile and other Francisella isolated from different species such as cod, tilapia, ticks and mammalian using sequences of $16 \mathrm{~s}$ rDNA gene. The phylogenetic tree was rooted with the $F$. tularensis cluster.

hipertróficas con vacuolas intracitoplasmáticas, algunas de las cuales contenían agrupaciones de bacterias cocoides basofílicas. En el tejido hematopoyético del bazo se observó una necrosis extensiva con degeneración y lisis de la membrana nuclear y citoplasmática. Los riñones se observaron con una glomeronefritis crónica y difusa con acumulación de centros de melanomacrófagos distribuidos ampliamente en el tejido necrótico. Hepatitis y necrosis ampliamente distribuida, con degeneración hidrópica de los hepatocitos y megalocitosis, con ruptura aislada de los ductos biliares presentando exudado biliar en el parénquima del hígado.
La tinción Gram permitió observar bacterias de formas cocoides y cocobacilares teñidas Gram negativas formando agrupaciones intracitoplasmáticas o libres en la matriz extracelular o detritus celular en diferentes tejidos como riñón, bazo, hígado, corazón, aletas y branquias.

El análisis por microscopia electrónica mostró agrupaciones de bacterias de forma cocoide y elongadas (300 a $1.000 \mathrm{~nm}$ ) dentro de vacuolas (endosomas o fagosomas) contenidas en el citoplasma de las células de las líneas celulares de tipo macrofágicas OKE-1 y SHK-1 (figura 3). 


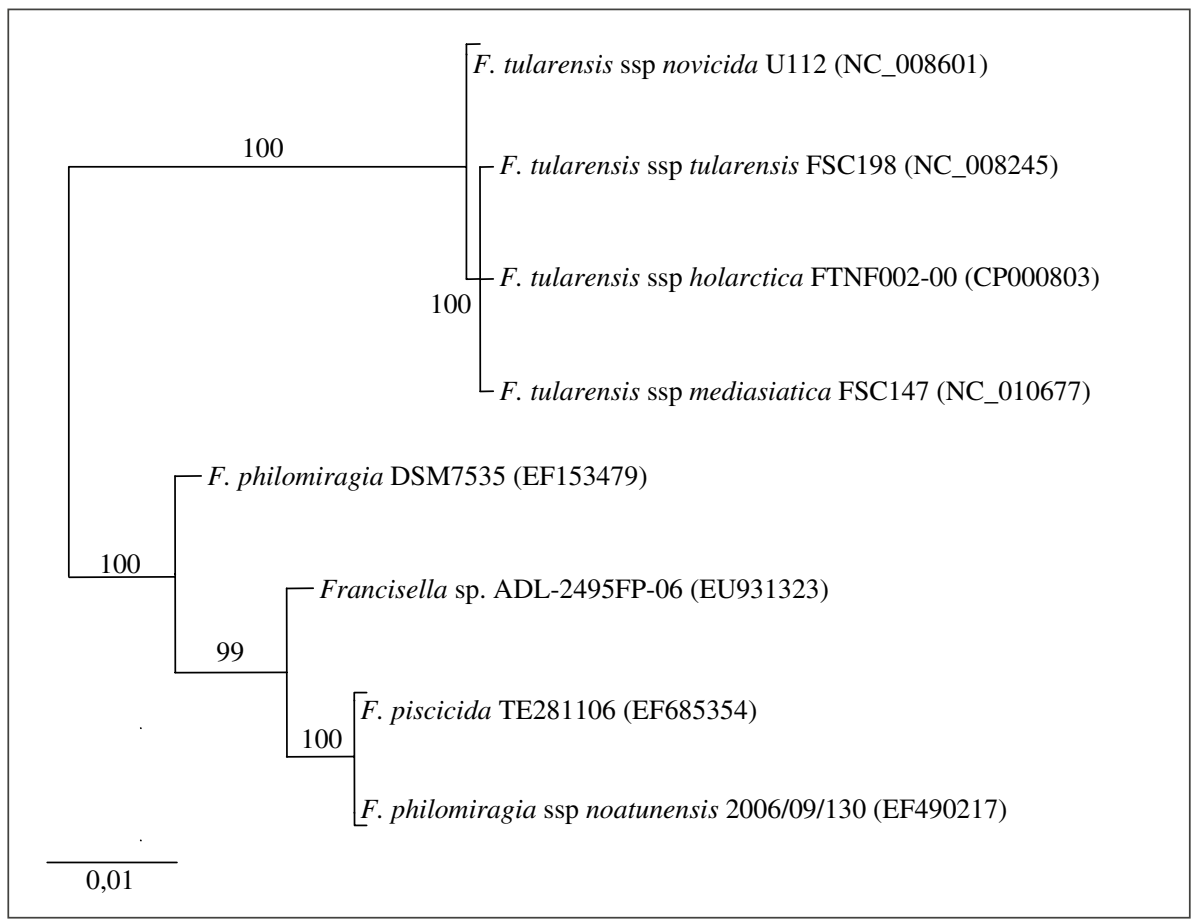

Figura 2a. Análisis filogenético entre los aislados de Francisella de salmón del Atlántico con otras Francisella usando las secuencias 16s+ITS+23s rDNA disponibles en GenBank.

Phylogenetic analysis between isolates of Francisella from Atlantic salmon and other Francisella using the sequences 16s+ITS+23s rDNA, available at GenBank.

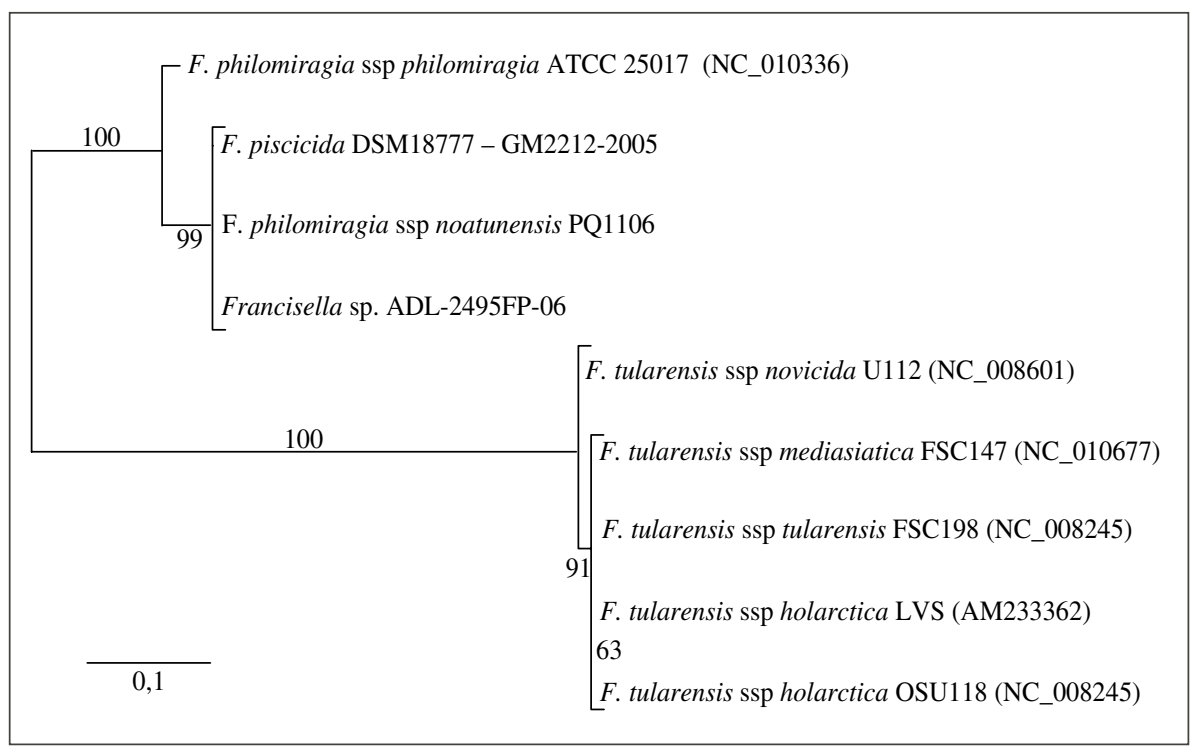

Figura 2b. Análisis filogenético multilocus entre los aislados de Francisella de salmón del Atlántico con otras Francisella usando las secuencias de los genes constitutivos $s d h A$, fopA, lpnB, groEL y rpoB.

Phylogenetic multilocus analysis between isolates of Francisella from Atlantic salmon and other Francisella using sequences of housekeeping genes $s d h A$, fopA, lpnB, groEL and rpoB. 


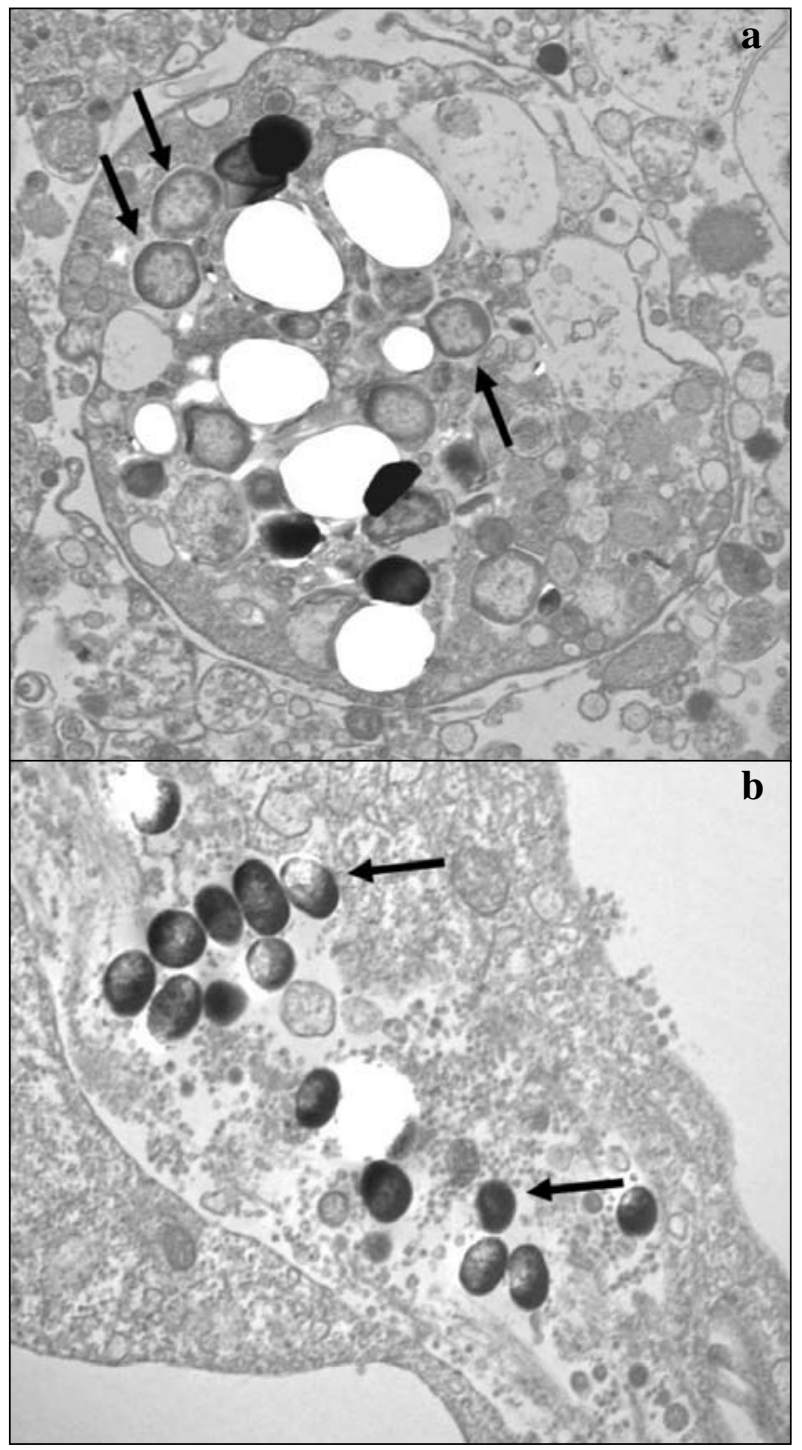

Figura 3. (a) Foto de microscopia electrónica de F. philomiragia aislados de salmón del Atlántico (flechas) formando agrupaciones dentro de un endosoma/fagosoma en el citoplasma de la línea celular OKE-1. (b) Estructuras cocoides y cocobacilares de las bacterias en el citoplasma de OKE-1

(a) Electronic microscopy photos of $F$. philomiragia isolated from Atlantic salmon (arrows) forming clusters inside an endosome/ phagosome in the cytoplasm of cell line OKE-1. (b) Cocoid and rod shaped structures of bacteria (arrows) in the cytoplasm of OKE-1.

\section{ANÁLISIS FILOGENÉTICO}

La secuencia nucleotídica del gen $16 s$ rDNA obtenida (número acceso: EU931323) del aislada fue analizada con el programa BLASTN y mostró 99\% de similitud con F. piscicida TU101106 (EF685355), F. philomiragia ssp noatunensis 2006/09/130 (EF490217), Francisella sp. AF-01-2 (AY928388) y F. philomiragia ssp philomiragia ATCC 25015 (AY928394) y un 97\% de similitud con F. tularensis ssp tularensis WY96-3418 (CP000608), F. tularensis ssp novicida U112 (CP000439), F. tularensis ssp mediasiatica FSC 147 (CP000915) y F. tularensis ssp holarctica FTNF002-00 (CP000803). El análisis filogenético de los genes $16 s+I T S+23 s$ rDNA del aislado mostró $100 \%$ de similitud con $F$. piscicida y $F$. philomiragia ssp noatunensis, 99\% similitud con $F$. philomiragia ssp philomiragia y $97 \%$ de similitud con las subespecies de F. tularensis. Finalmente, el análisis multilocus de los genes constitutivos mostró $100 \%$ de similitud con $F$. piscicida y F. philomiragia ssp noatunensis, un $96 \%$ de similitud con F. philomiragia ssp philomiragia y un $84 \%$ de similitud todas las subespecies de F. tularensis.

\section{DISCUSIÓN}

Salmón del Atlántico cultivado en el lago Llanquihue presentó, sin precedentes en la acuicultura nacional, altas tasas de morbilidad y mortalidad por la bacteria entre junio (Birkbeck y col 2007) a diciembre del año 2006, con un comportamiento, signos y lesiones muy similares a las descritas por organismos similares a U2 (Birkbeck y col 2007), otras rickettsias (RLO) y a Francisella sp. detectadas en otras partes del mundo, como en tilapia en Costa Rica (Mauel y col 2007) y Hawaii (Mauel y col 2003), bacalao en Noruega (Nylund y col 2006, Ottem y col 2007 ), corvina blanca en California (Chen y col 2000), mero en Taiwán (Chen y col 1994) y U2 en puyes en Chile (Enríquez y col 1998).

Una inusual alza en la densidad de puyes (Galaxias maculatus) dentro y fuera de las balsas-jaulas con altos niveles de $F$. philomiragia en riñón, hígado y bazo (datos no mostrados), más la evidencia de transmisión horizontal entre el puye y salmón del Atlántico (Brossard 1999) en el caso de tratarse de U2, podría explicar la rápida diseminación en poco tiempo y la persistencia de los cuadros clínicos en casi todos los centros de cultivo de salmón del Atlántico presentes en el lago Llanquihue. Estos sucesos también han sido descritos en otros RLOs y Francisella sp., donde la transmisión horizontal es por contacto directo entre peces de la misma especie o diferentes especies o por medio de la misma agua (Chern y Chao 1994, Chen y col 1994, Mauel y col 2003) y en una amplia gama de peces de agua dulce y mar (Mauel y col 2007) y de temperaturas que van entre los $6^{\circ} \mathrm{C}$ hasta los $26^{\circ} \mathrm{C}$ (Nylund y col 2006, Mauel y col 2007).

El análisis histopatológico reveló un patrón muy similar a los ya descritos previamente en Chile en salmón del Atlántico afectados por U2 (Cvitanich y col 1995), con lesiones de tipo granulomatosas de distribución difusa y penetrantes en los diferentes tejidos del huésped con un tropismo por los fagocitos o células que cumplen función fagocítica, muy similar a lo observado por análisis histopatológicos descritos en otras especies como bacalao (Nylund y col 2006) y tilapia (Mauel y col 2003) afectados por Francisella. El análisis ultraestructural de la bacteria mostró un tamaño de $1 \mu \mathrm{m}$ en su forma elongada, muy similar a lo observado en $F$. philomiragia con un tamaño menor a $1,5 \mu \mathrm{m}$ pero 
Cuadro 2. Cambios nucleotídicos de los genes $16 s+I T S+23 s$ rDNA según las diferentes subespecies de Francisella. Secuencia de referencia (posición 1 a 1840), número de acceso GenBank DQ309246.

Nucleotides changes of the genes $16 s+I T S+23 s$ rDNA in different subspecies of Francisella. Reference sequence (Position 1 to 1840) accession code DQ309246.

\begin{tabular}{|c|c|c|c|c|c|c|c|c|c|c|c|}
\hline \multirow{2}{*}{ Especies bacterianas } & \multirow{2}{*}{ Huésped } & \multicolumn{10}{|c|}{ Posición (1-1840 16s+ITS+23s, referencia DQ309246) } \\
\hline & & $166-191$ & 215 & $431-433$ & 550 & 1557 & 1589 & 1594 & $1673-1684$ & 1726 & 1823 \\
\hline F. philomiragia & Salar & CTTTGT & $\mathrm{G}$ & GTA & A & A & G & A & ---GTAATT--- & $\mathrm{C}$ & A \\
\hline F. philomiragia ssp noatunensis & Bacalao & CTTTGT & A & GTA & G & G & A & G & ---GTAATT--- & $\mathrm{T}$ & $\mathrm{T}$ \\
\hline F. philomiragia & Tilapia & CTTTGT & $\mathrm{G}$ & GCA & G & A & A & G & ---GTAATT--- & $\mathrm{C}$ & A \\
\hline F. philomiragia ssp philomiragia & Ratas & --TTAG & G & GTA & G & A & A & G & ---GTAATT--- & $\mathrm{C}$ & A \\
\hline F. Tularensis ssp tularensis & Humanos & TTTCGG & G & TGG & G & A & A & G & TTTTAGGTTTAG & $\mathrm{C}$ & A \\
\hline F. Tularensis ssp novicida & Humanos & TTTCGG & G & TGG & G & A & A & G & TTTTAGGTTTAG & $\mathrm{C}$ & A \\
\hline F. Tularensis ssp holarctica & Humanos & TTTCGG & G & TGG & $\mathrm{G}$ & A & A & G & TTTTAGGTTTAG & $\mathrm{C}$ & A \\
\hline F. Tularensis ssp mediasiatica & Humanos & TTTCGG & $\mathrm{G}$ & TGG & $\mathrm{G}$ & A & A & $\mathrm{G}$ & TTTTAGGTTTAG & $\mathrm{C}$ & A \\
\hline
\end{tabular}

Cuadro 3. Características fenéticas entre la diferentes subespecies y orígenes de Francisella. $F$. philomiragia $($ Chile $=$ salmón del Atlántico, Noruega = bacalao del Atlántico, tilapia = Asia y Centroamérica) y F. philomiragia $\operatorname{ssp}$ philomiragia con $F$. tularensis $=$ especie tipo.

Phenetic characteristics between different subspecies and origins of Francisella. F. philomiragia $($ Chile $=$ Atlantic salmon, Norway $=$ Atlantic cod, tilapia $=$ Asia and Center of America) y F. philomiragia $\operatorname{ssp}$ philomiragia with F. tularensis $=$ reference strain.

\begin{tabular}{cccccc}
\hline Característica & Chile & Noruega & Tilapia & F. philomiragia & F. tularensis \\
\hline Temperatura & 22 & 22 & nd & 37 & 37 \\
TSA & No & Sí, Lento & No & Sí & Sí \\
BHI & No & Sí, Lento & No & Sí & Sí \\
Oxidasa & Negativo & Negativo & nd & Positivo & Positivo \\
$\mathrm{NaCl} 6 \%$ & No & Sí & nd & No & No \\
\hline
\end{tabular}

diferente a $F$. tularensis (Nylund y col 2006, Ottem y col $2007^{\mathrm{a}}$ ) que generalmente es menor a $0,5 \mu \mathrm{m}$.

Por otra parte, una diferencia importante de los aislados bacterianos de salmón del Atlántico es que no crecen en medios con concentración de cloruro de sodio por sobre $2 \%$, a diferencia de F. piscicida (Nylund y col 2007) o F. philomiragia ssp noatunensis (Mikalsen y col 2007) aislados de bacalao en Noruega y que crecen en agar nutriente al $6 \% \mathrm{NaCl}\left(\right.$ Ottem y col 2007 ${ }^{\mathrm{a}}$ ); esto explica que la Francisellosis en bacalao se exprese en agua de mar sin problemas y también podría explicar que la enfermedad en salmón del Atlántico solo se exprese en agua dulce y que solo haya sido aislado, detectado en estuario y mar pero sin la expresión de la enfermedad. Otro antecedente relevante es el desarrollo óptimo de los aislados bacterianos entre 18 a $22{ }^{\circ} \mathrm{C}$ y un menor desarrollo a temperaturas inferiores a $15^{\circ} \mathrm{C}$, lo cual podría explicar el aumento en la incidencia de los casos durante la época de temperaturas más elevadas o años más calurosos.

Desde el punto de vista zoonótico, no se han registrado casos de infecciones accidentales en humanos de F. philomiragia aisladas de salmón del Atlántico o por las Francisella sp. aisladas desde peces en otras partes del mundo. Esto podría estar relacionado con la incapacidad que tienen estas fenovariantes de $F$. philomiragia de crecer a temperaturas más altas como $37^{\circ} \mathrm{C}$, a diferencia de F. philomiragia ssp philomiragia y todas las subespecies de
F. tularensis que crecen muy bien a $37^{\circ} \mathrm{C}$ y que provocan enfermedades infecciosas en humanos y otros mamíferos terrestres. Las características fastidiosas en crecimiento de aislados bacterianos de salmón del Atlántico en líneas celulares y medios basados en agar o caldo son muy similares a las encontradas en $F$. piscicida (Nylund y col 2006) o F. philomiragia ssp noatunensis (Mikalsen y col 2007); sin embargo, son diferentes a $F$. philomiragia ssp philomiragia y las subespecies de $F$. tularensis que crecen en condiciones nutricionales mucho menos exigentes y en menor tiempo o diferentes incluso a las Francisella sp. detectadas en tilapia, las cuales no han podido ser aisladas tanto en líneas celulares como en medios basados en agar (Mauel y col 2007).

Desde el punto de vista filogenético, el análisis del gen $16 s$ rDNA (figura 1 y 2 a) y de los genes constitutivos (figura 2b) mostraron que los aislados bacterianos de salmón del Atlántico pertenecen al grupo de las $F$. philomiragia, al igual que las Francisella sp. detectadas desde bacalao en Noruega o en tilapia en Costa Rica y Asia. El grupo $F$. philomiragia presenta dos subespecies definidas por el comité internacional de taxonomía y que corresponde a la especie tipo $F$. philomiragia quedando como $F$. philomiragia ssp philomiragia y $F$. noatunensis ${ }^{12}$ (F. piscicida) quedando como F. philomiragia ssp noatunensis.

\footnotetext{
12 Mikalsen J, 2008. Comunicación personal.
} 
El análisis del alineamiento múltiple de secuencias reveló que en el ITS ubicado entre el gen $16 s$ y $23 s$ rDNA existe una zona comprendida entre la posición 1673 a la 1684 que diferencia claramente (cuadro 2) entre las especies tipo $F$. philomiragia de la $F$. tularensis; sin embargo, existen otras regiones como la comprendida entre la posición 166 a la 191 (cuadro 2) que diferencian $F$. philomiragia ssp noatunensis de $F$. philomiragia ssp philomiragia de ratas (Ottem y col $2007^{\mathrm{b}}$ ) y de las subespecies de F. tularensis. Más aún, otras posiciones como las 215, 1557, 1726, 1823 (cuadro 2) diferencian F. philomiragia de salmón del Atlántico con la firma o combinación "AGTT" de todo el resto de las especies de Francisella y que poseen "GACA" (cuadro 2) en esas mismas posiciones. Sin embargo, e independiente de las variaciones nucleotídicas encontradas dentro del grupo F. philomiragia (cuadro 2), el análisis filogenético (figura 1, 2a y 2b) reveló que las Francisellas aisladas en bacalao en Noruega, salmón del Atlántico en Chile y tilapia en Centroamérica y Asia, pertenecerían al mismo grupo de F. philomiragia ssp noatunensis (Mikalsen y col 2007), aunque fenéticamente las Francisellas aisladas en estos diferentes huéspedes, incluyendo salmón del Atlántico, presentan características propias diferentes a las encontradas entre los miembros de la misma subespecie (cuadro 3), por lo cual es necesario hacer más investigaciones filogenéticas con nuevos marcadores o filogenómica.

\section{RESUMEN}

Francisella philomiragia fue aislada de salmón del Atlántico cultivado en balsas-jaulas en el lago Llanquihue con brotes de una enfermedad granulomatosa con altas tasas de morbilidad y mortalidad acumuladas entre $5 \%$ a $20 \%$. Los aislados bacterianos tienen $100 \%$ similitud con $F$. philomiragia ssp noatunensis o F p piscicida aislado de bacalao en Noruega, 99\% de similitud con Francisella sp. detectado en tilapia en Asia y Centroamérica y $99 \%$ de similitud con la especie tipo $F$. philomiragia por análisis filogenético del gen $16 \mathrm{~s}$ rDNA.

\section{REFERENCIAS}

Barns SM, CC Grow, RT Okinaka, P Keim, CR Kuske. 2005. Detection of diverse new Francisella-like bacteria in environmental samples. Appl Environ Microbiol 71, 5494-5500.

Birkbeck TH, M Bordevik, MK Froystad, A Baklien. 2007. Identification of Francisella sp. From Atlantic salmon, Salmo salar L., in Chile. J Fish Dis 30, 505-507.

Bravo S, M Campos. 1989. Síndrome del salmón Coho. Chile Pesquero 54, 47-48.

Brossard MA. 1999. Determinación experimental de la vía de contagio del agente U2 (RLO) aislado de puye (Galaxias maculatus) en salmón del Atlántico (Salmo salar). Memoria de título, Escuela de Medicina Veterinaria, Universidad Austral de Chile, Valdivia, Chile.

Chen SC, MC Tung, SP Chen, JF Tsai, PC Wang, RS Chen, SC Lin, A Adams. 1994. Systematic granulomas caused by a rickettsia-like organism in Nile tilapia, Oreochromis niloticus (L.), from Southern Taiwan. J Fish Dis 17, 591-599.

Chen MF, S Yun, GD Marty, TS McDowell, ML House, JA Appersen, TA Guenther, KD Arkush, RP Hedrick. 2000. A Piscirickettsia-like bacterium associated with mortality of white seabass Atractoscion nobilis. Dis Aquat Org 43, 117-126.
Chen SC, PC Wang, MC Tung, KD Thompson, A Adams. 2000b. A Piscirickettsia like organism in grouper, Epinephelus melanostigma, in Taiwan. J Fish Dis 23, 415-418.

Chern RS, CB Chao. 1994. Outbreaks of a disease caused by a rickettsia-like organism in cultured tilapias in Taiwan. Fish Pathol 29, 61-71.

Cvitanich J, O Gárate, C Silva, M Andrade, C Figueroa, C Smith. 1995. Isolation of a new rickettsia-like organism from Atlantic salmon in Chile. FHS/AFS newsletter 23, 1-3.

Enríquez R, M Monras, A Ceballos, C Igor. 1998. Primer aislamiento de un organismo rickettsial desde Galaxias maculatus (puye). Arch Med Vet 30, 233-234.

Fryer JL, CN Lannan, LH Garces, JJ Larenas, PA Smith. 1990. Isolation of a rickettsiales-like organisms from disease Coho Salmon (Oncorhyncus kisutch) in Chile. Fish Pathol 25, 107-114.

Fryer JL, CN Lannan, SJ Giovannoni, ND Word. 1992. Piscirickettsia salmonis gen. nov., sp. nov., the causative agent of a epizootic disease in salmonid fishes. Int J Syst Bact 42, 120-126.

Higgins DG, PM Sharp. 1988. CLUSTAL: a Package for Performing Multiple Sequence Alignment in a Microcomputer. Gene 73, 237-244.

Hollis DG, RE Weaver, AG Steigerwalt, JD Wenger, CW Moss, DJ Brenner. 1989. Francisella philomiragia comb. nov. (formerly Yersinia philomiragia) and Francisella tularensis biogroup novicida (formerly Francisella novicida) associated with human disease. J Clin Microbiol 27, 1601-1608.

Jensen WI, CR Owen, WL Jellison. 1969. Yersinia philomiragia sp.n., a new member of the Pasteurella group of bacteria, naturally pathogenic for the muskrat (Ondatra Zibethica). J Bacteriol 100, 1237-1241.

Kimura M. 1980. A simple method for estimating evolutionary rates of base substitutions through comparative studies of nucleotide sequences. J Mol Evol 16, 111-120.

Mauel MJ, DL Miller, K Frazier, AD Liggett, L Styer, D MontgomeryBrock, J Brock. 2003. Characterization of Piscirickettsiosis-like disease in Hawaiian tilapia. Dis Aquat Org 53, 249-255.

Mauel MJ, E Soto, JA Moralis, J Hawke. 2007. A Piscirickettsiosislike syndrome in cultured tilapia (Oreochromis niloticus) in Latin America with Francisella sp. as the pathogenic agent. J Aquat Anim Health 19, 27-34

Mikalsen J, AB Olsen, T Tengs, DJ Colquhoun. 2007. Francisella philomiragia subsp. noatunensis subsp. nov., isolated from framed Atlantic cod (Gadus morhua L.). Int J Syst Evol Microbiol 57, 1960-1965.

Nano FE. 1992. Francisella. In: Balows A, Truëper HG, Sworkin M, Harder W, Schleifer KH (eds). The Prokaryotes. Springer-Verlag, New York, USA Pp 3987-3993.

NCCLS. 2003. Methods for broth dilution susceptibility testing of bacteria isolated from aquatic animals; approved guideline. NCCLS M49-A. 940 West Valey Road, Suite 1400, Wayne, Pennsylvania, USA.

Nylund A, KF Ottem, K Watanabe, E Karlsbakk, B Krossøy. 2006. Francisella sp. (Family Francisellaceae) causing mortality in Norwegian cod (Gadus morhua) farming. Arch Microbiol 185, 383-392

Ottem KF, A Nylund, E Karlsbakk, A Friis-Møller, B Krossøy. 2007ª. Characterization of Francisella sp., GM2212, the first Francisella isolate from marine fish, Atlantic cod (Gadus morhua) Arch Microbiol 187, 343-350

Ottem KF, A Nylund, E Karlsbakk. 2007 . New species in the genus Francisella (Gammaproteobacteria; Francisellaceae); Francisella piscicida sp. Nov. isolated from cod (Gadus morhua). Arch Microbiol 188, 547-550.

Scoles GA. 2004. Phylogenetic analysis of the Francisella-like endosymbionts of Dermacentor ticks. J Med Entomol 41, 277286.

Sjøstedt AB. 2005. Family III. Francisellaceae fam. nov. In: Brenner DJ, Krieg NR, Staley JT (eds). Bergey's manual of systematic bacteriology. The gamma-proteobacteria. $2^{\text {nd }}$ ed. Vol II, Part B. Springer, New York, USA, Pp 199-200. 